\title{
Transcription in tight spaces
}

n the first direct comparison between the spatial organization of a region of the human genome with the syntenic region in the mouse, Mahy et al. report on page 579 that genes are not preferentially localized to the periphery of chromosome territories. The results contradict earlier analyses of nuclear organization, and suggest that a popular model correlating chromosome territory organization with transcriptional activity may need to be revised.

Previous studies have suggested that genes lie on the chromosome territory periphery, whereas intergenic sequences occupy the interior of the territory. According to the inter-chromosome domain (ICD) model, this is because the higher order structure of the territory blocks access by the transcriptional machinery. The authors compared the spatial organization of a 1-Mb stretch of the human genome and the syntenic murine sequence, and found that although there is a conserved spatial organization within these territories, genes in these segments do not preferentially localize to the periphery. Using cell lines with different gene expression patterns, Mahy et al. also saw no differences in the intraterritory positions of expressed and repressed genes, suggesting that the transcriptional machinery can reach genes without gross remodeling of chromosomal territories.
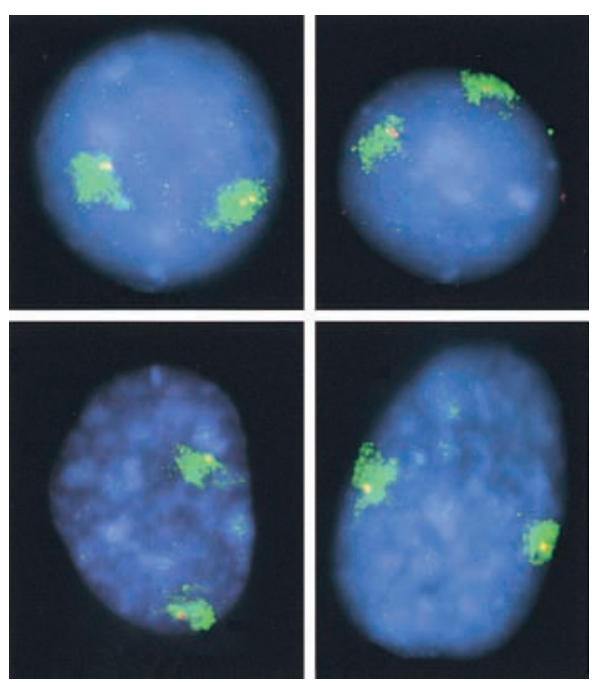

Genes are expressed (red) from varying sites in chromosome territories (green).

\section{A muscular job for spectrin}

\begin{abstract}
11 multicellular animals examined have a spectrin
cytoskeleton, suggesting that it serves some important, although so far poorly understood, function. On page 665 , Norman and Moerman provide significant new insights into spectrin activity in $C$. elegans, where the tetrameric molecule seems to be required for normal development of body wall muscle and muscle attachment structures.

The authors identified and characterized $s p c-1$, the only $\alpha$ spectrin gene in $C$. elegans. Localization of $\alpha$ spectrin to the cell membrane during development requires $\beta$ spectrin, but not $\beta_{\text {Heavy }}$ spectrin. A null mutation in spc-1 blocks complete elongation of the worm embryo, which dies shortly after hatching. Elongation appears to be required for normal muscle development, and worms with mutations in spectrin genes develop a disorganized apical actin cytoskeleton in the hypodermis and broad muscle cells with misshapen myofilaments.
\end{abstract}

Surprisingly, the absence of spectrin does not appear to cause the broad muscle cell shape directly. Instead, the absence of spectrin in

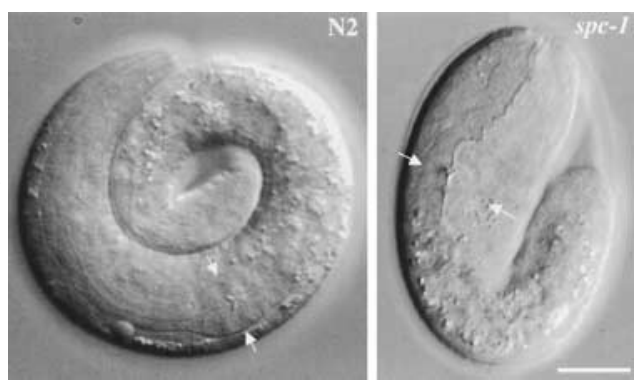

Worms lacking $\alpha$-spectrin (right) do not elongate. thehypodermis interferes with microfilament stability, which in turn interferes with the early elongation of the embryo. Broad muscle cells are a result of this failure in elongation. The authors' new genetic system should facilitate further dissection of complex interactions between tissues during development.

\section{Arresting development}

n meiosis II, vertebrate oocytes undergo a cell cycle arrest that is maintained until fertilization, but it has been difficult to identify the factors responsible for this process. By screening a novel cDNA library, Lefebvre et al., page 603, have discovered and characterized a protein that specifically regulates meiosis II spindle integrity during arrest. The work helps to explain how the cell distinguishes between meiosis I and II, and the new library should be useful in discovering additional genes involved in early development.

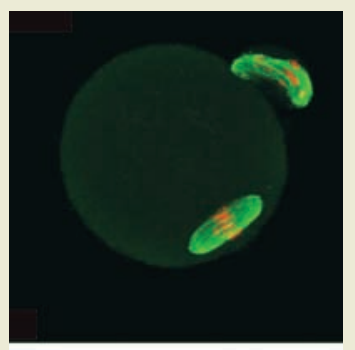

Immature oocytes do not initiate an arrest until they reach meiosis II, but the kinases known to trigger this process are expressed through both meiotic divisions, so other factors must determine how and when the arrest occurs. The authors generated a cDNA library from mouse immature oocytes, representing transcripts that are

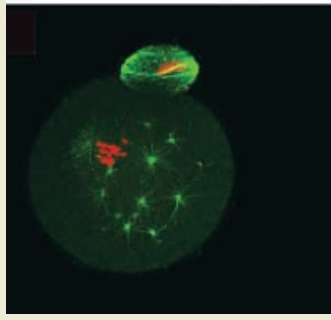

MISS depeletion (bottom) causes defects in meiosis II spindles. specifically relevant to meiotic maturation. Two-hybrid screening identified a MAP kinase-interacting and spindle-stabilizing protein (MISS). MISS is unstable in meiosis I, but becomes stable in meiosis II, when it localizes in dots on the spindles. Interfering with endogenous MISS RNA causes severe spindle defects only in meiosis II. Its specific stabilization during meiosis II suggests that MISS provides a link between MAP kinase activity and meiotic arrest. 